\title{
Carcinoembryonic Antigen Positive
}

National Cancer Institute

\section{Source}

National Cancer Institute. Carcinoembryonic Antigen Positive. NCI Thesaurus. Code C128808.

A laboratory test result indicating the presence of carcinoembryonic antigen in a sample. 